surface potential during phagocytosis. Science. 2006;313(5785):347-351.

17. Michaelson D, et al. Rac1 accumulates in the nucleus during the G2 phase of the cell cycle and promotes cell division. J Cell Biol. 2008;181(3):485-496.

18. Sung PJ, Rodrigues AB, Kleinberger A, Quatela S, Bach EA, Philips MR. Cytosolic ras supports eye development in Drosophila. Mol Cell Biol. 2010;30(24):5649-5657.

19. Reid TS, Terry KL, Casey PJ, Beese LS. Crystallographic analysis of CaaX prenyltransferases com- plexed with substrates defines rules of protein substrate selectivity. J Mol Biol. 2004;343(2):417-433.

20. Bos JL, et al. The role of Rap1 in integrin-mediated cell adhesion. Biochem Soc Trans. 2003;31(pt 1):83-86.

21. Li Y, et al. Rap1a null mice have altered myeloid cell functions suggesting distinct roles for the closely related Rap1a and $1 \mathrm{~b}$ proteins. J Immunol. 2007;179(12):8322-8331.

22. Cope AP, Schulze-Koops H, Aringer M. The central role of T cells in rheumatoid arthritis. Clin Exp Rheumatol. 2007;25(5 suppl 46):S4-S11.
23. Whyte DB, et al. K- and N-Ras are geranylgeranylated in cells treated with farnesyl protein transferase inhibitors. J Biol Chem. 1997;272(22):14459-14464.

24. Kazi A, et al. Blockade of protein geranylgeranylation inhibits Cdk2-dependent p27Kip1 phosphorylation on Thr187 and accumulates p27Kip1 in the nucleus: implications for breast cancer therapy. $\mathrm{Mol}$ Cell Biol. 2009;29(8):2254-2263.

25. Philips MR, Cox AD. Geranylgeranyltransferase I as a target for anti-cancer drugs. J Clin Invest. 2007;117(5):1223-1225.

\title{
Navigational error in the heart leads to premature ventricular excitation
}

\author{
Hiroshi Akazawa and Issei Komuro
}

Department of Cardiovascular Medicine, Osaka University Graduate School of Medicine, Osaka, Japan.

\begin{abstract}
In the normal heart, an insulating barrier separates the atria and ventricles. The only way in which electrical impulses can cross this barrier is via the atrioventricular (AV) node, which delays impulse conduction to ensure the forward flow of the blood. However, in some individuals, additional muscular bundles (accessory pathways) allow rapid conduction of electrical impulses from the atria to the ventricles, resulting in premature ventricular excitation and contraction. In this issue of the JCI, two independent research groups demonstrate that erroneous development of the embryonic AV canal, which performs a similar function to that of the adult $\mathrm{AV}$ node, is a novel mechanism by which accessory pathways can form.
\end{abstract}

Three-dimensional organization of the electrical conduction system allows the heart to generate rhythmic forward flow of the blood (Figure 1A). After triggering atrial contraction, the electrical impulses are delayed at the atrioventricular (AV) junction for an optimal period of time that allows the ventricles to fill up with blood before ventricular contraction is initiated. In adult hearts, this delay is attributable to slow conduction by specialized cardiomyocytes in the AV node. After this delay, the electrical impulses rapidly travel to the ventricular chamber myocardium via the His-Purkinje system and stimulate ventricular contraction. The AV node/His-bundle pathway is the only muscular connection between the atrial and ventricular chambers, which are electrically insulated from each other by the annulus fibrosus, a ring-like struc-

Conflict of interest: Issei Komuro has received research support from Takeda Pharmaceutical Co. Ltd., Daiichi Sankyo Co. Ltd., MSD K.K., Shionogi \& Co. Ltd., Nippon Boehringer Ingelheim Co. Ltd., Astellas Pharma Inc., Novartis Pharma K.K., and Kowa Pharmaceutical Co. Ltd.

Citation for this article: J Clin Invest. doi:10.1172/ JCI46038. ture that consists of connective tissues impermeable to electrical propagation.

Development of the AV conduction system involves a precisely coordinated process of cellular differentiation and multicellular morphogenesis. Therefore, even subtle perturbation of the developmental process can give rise to anatomical or functional defects of the cardiac conduction system and thereby causes serious arrhythmias. For example, in patients with Wolff-Parkinson-White (WPW) syndrome, in addition to the AV node/His-Purkinje system pathway, extra electrically active muscular connections known as accessory pathways exist between the atria and ventricles. Electrical impulses travel via accessory pathways and induce earlier contraction of the ventricular myocardium (preexcitation) than do electrical impulses traveling via the $\mathrm{AV}$ node/His-Purkinje system. In this situation, antegrade $A V$ nodal conduction may immediately trigger retrograde conduction through the accessory pathways and re-excite the atria, leading to initiation and perpetuation of reentrant tachycardia. Even more ominous is the rapid and preexcited ventricular response to atrial fibrillation in patients with WPW syndrome, which can precipitate life-threatening ventricular fibrillation. Despite the clinical importance of ventricular preexcitation, the underlying molecular mechanisms have not been well understood. In this issue of the JCI, two independent research groups report genetic approaches in mice that shed light on the mechanisms for accessory pathway formation and ventricular preexcitation $(1,2)$. Specifically, the data from both groups support the notion that missteps during development of the $\mathrm{AV}$ canal give rise to ventricular preexcitation.

\section{Defective formation of the annulus fibrosus}

Molecular insights into the mechanisms underlying ventricular preexcitation were not elucidated until a candidate gene for a familial form of WPW syndrome was identified $(3,4)$. In two unrelated families with WPW syndrome accompanied by hypertrophic cardiomyopathy, a dominant missense mutation $(\mathrm{R} 302 \mathrm{Q})$ was observed in the gene that encodes the $\gamma 2$ regulatory subunit of AMP-activated protein kinase (AMPK), PRKAG2 (3, 4). Subsequent biochemical and histological studies indicated that PRKAG2 mutations affect the basal activity of AMPK, a "fuel gauge" participating in the regulation of energy homeostasis (5). Transgenic mice overexpressing human PRKAG2 with a WPW syndrome-causing mutation, such as R302Q or N488I, in the heart, showed cardiac glycogen storage and manifested ventricular hypertrophy and preexcitation $(6,7)$. Strikingly, histopathology of the hearts revealed that the annulus fibrosus was disrupted by intercalation of glycogen-loaded cardiomyocytes. These studies have impli- 
A Normal heart

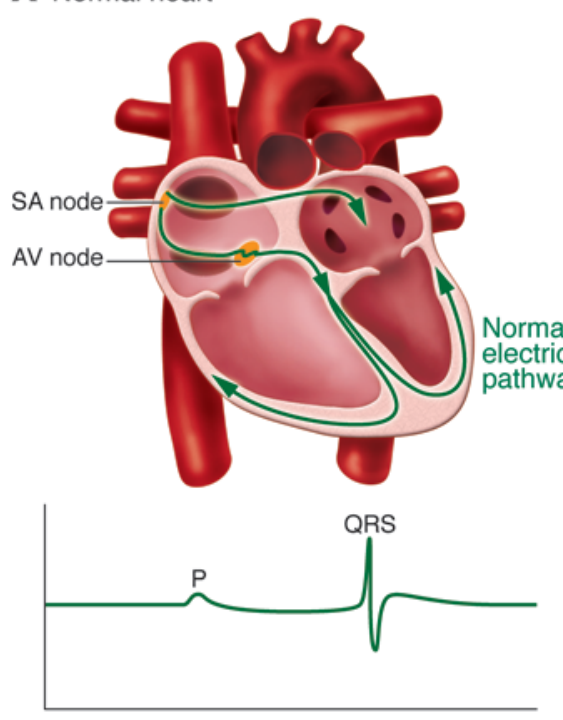

C Notch1 activated

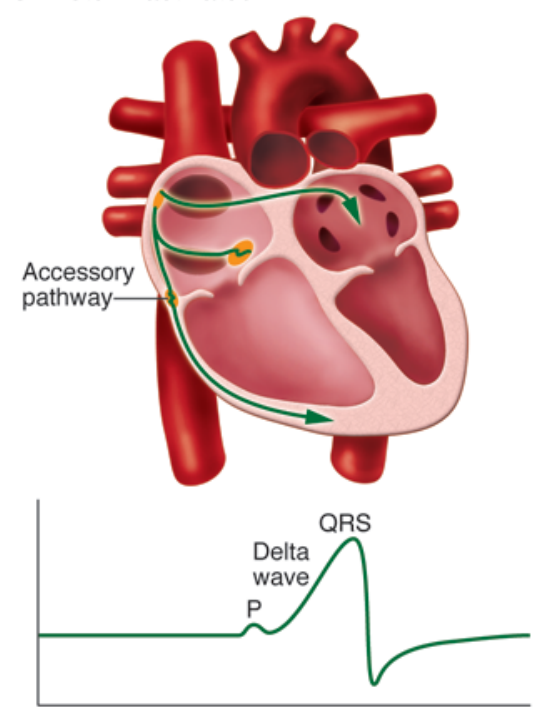

cated disruption of the annulus fibrosus by cardiac glycogen accumulation as a cause of ventricular preexcitation in WPW syndrome associated with PRKAG2 mutations.

These data, indicating that intercalation of glycogen-loaded cardiomyocytes disrupts the annulus fibrosus in patients with a familial form of WPW syndrome associated with PRKAG2 mutations, suggest that in other forms of WPW syndrome, the formation of accessory pathways may originate from failure of the annulus fibrosus to fully insulate the atrial and ventricular chambers. In the developing human heart, the sulcus tissue (located at the epicardial side) invaginates inward and fuses with the endocardial AV cushion, thereby creating

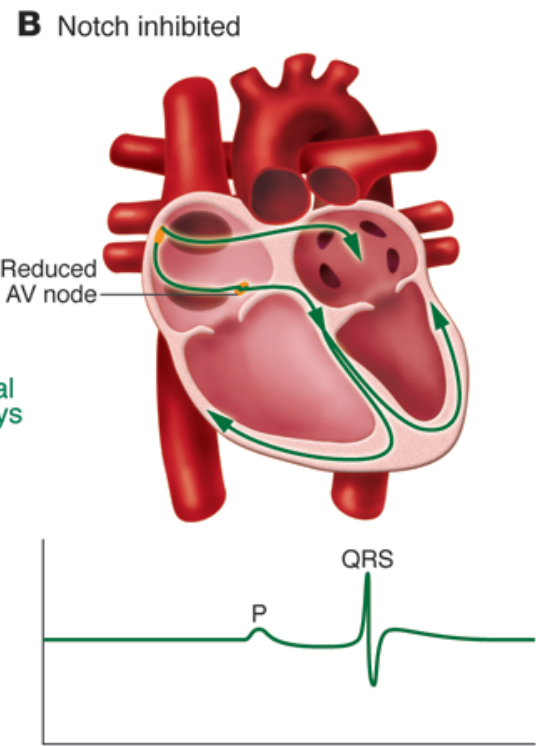

D Tbx2 deficiency
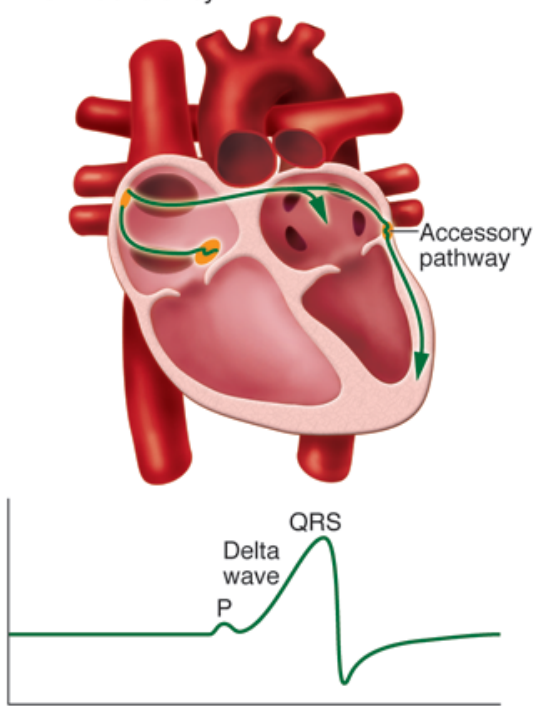

the insulation between the atria and ventricles (8). A cluster of cells, termed epicardium-derived cells (EPDCs), migrates through the AV junctional myocardium to populate the endocardial AV cushion (9), and surgical interruption of EPDC migration results in the production of broad bundles of accessory pathways and overt ventricular preexcitation in quail embryos (9). Collectively, the studies discussed here (in Defective formation of the annulus fibrosus) led to the hypothesis that defective formation of the annulus fibrosus was a possible mechanism underlying the formation of accessory pathways, but it remains unclear how annulus fibrosus malformation affects conductance of the accessory pathways.

\section{Figure 1}

Cardiac conduction in normal, Notch-inhibited, Notch1-activated, and Tbx2-deficient mice. (A) In normal hearts, the electric impulses initiated by pacemaker cells in the sinoatrial (SA) node propagate through the atrial myocardium and trigger its contraction. At the AV node, the impulses are delayed for a period to facilitate alternating contraction of the atrial and ventricular myocardium. After the AV delay, the electrical impulses rapidly travel to the ventricular myocardium via the HisPurkinje system and stimulate the ventricular myocardium. (B) In Notch-inhibited hearts, the AV node is hypoplastic, and the PR interval on the ECG is shortened due to disruption of the AV nodal delay. (C) In Notch1-activated and (D) Tbx2-deficient hearts, accessory pathways are formed as a result of malformation of the AV canal myocardium. The accessory pathways are commonly right-sided in Notch1-activated mice and left-sided in Tbx2deficient mice. Because of faster conduction through the accessory pathways than through the AV node, the ventricular myocardium is prematurely stimulated (preexcitation). The ECG shows a short PR interval, a slurred upstroke ("delta wave") of the QRS complex, and a widened QRS complex.

\section{Embryonic remnants of the AV canal} It is justifiable to presume that accessory pathways may form as a sequela of malformation of the cardiac conduction system at the $\mathrm{AV}$ junction. After the formation of the primitive heart tube during early embryonic development, the AV canal myocardium develops between the atrial and ventricular myocardium $(10,11)$. The AV canal myocardium gives rise to several cardiac components, including the future AV valves, ventricular septum, outflow tract, and atrial part of the AV conduction axis (12). In addition, because of its slow conduction property, AV canal myocardium, much like the AV node in the adult, contributes to an effective delay between contraction of the 
atrium and ventricle during embryogenesis (13). While the continuity of the AV myocardium gradually disappears as formation of the annulus fibrosus progresses, remnant strands of AV myocardium are still observed morphologically, even in normal perinatal hearts of quails and humans (14, 15). These remnant strands may lead to preexcitation and serve as a substrate for reentrant tachycardia affecting neonates, but shortly thereafter, most tachycardias resolve spontaneously, as the remnant strands lose their electrical conductivity $(14,15)$. These morphological and electrophysiological observations raise the possibility that accessory pathways might be embryonic remnants derived from the AV canal that acquire fast conduction properties by some as yet undefined mechanisms. However, experimental evidence for this hypothesis has been lacking to date.

\section{When navigation goes awry in the AV canal}

In this issue of the JCI, two independent research groups have provided experimental evidence in mice that ventricular preexcitation arises as a consequence of aberrant Notch signaling and T-box 2 transcription factor (Tbx2) function, both of which play a regulatory role in the normal patterning and functional maturation of the AV canal myocardium $(1,2)$. The genetic blueprint for AV canal development is just emerging. During early gestation in the chick and mouse, the Notch and Hairy-related transcription factors are expressed in adjacent atrial and ventricular chamber myocardium and restrict expression of bone morphogenetic protein 2 (BMP2) and $\mathrm{Tb} 2 / 3$ to the AV canal myocardium (16, 17). Tbx2 is a downstream target of the BMP2 signaling pathway (18), and Tbx2 and Tbx3 are coexpressed and functionally redundant (10).

In this issue of the JCI, Rentschler and colleagues demonstrate that inhibition of Notch signaling by transgenic expression of a truncated form of the protein mastermind-like (MAML) in the ventricular myocardium and AV canal results in hypomorphic formation of the AV node, with defective AV nodal delay in mice (Figure $1 \mathrm{~B}$ and ref. 1). In contrast, activation of Notch signaling by transgenic expression of the Notch1 intracellular domain in ventricular myocardium and AV canal led to formation of epicardial and right-sided muscular accessory pathways and expansion of AV nodal tissue in postnatal mice (Figure 1C and ref. 1). As a result, Notch1-activated mice manifested ventricular preexcitation postnatally. Interestingly, the AV refractory period in Notch1-activated mice was short, and they were predisposed to atrial tachycardia upon programmed electrical stimulation. Despite unchanged expression of BMP2 in the AV canal, Notch1-activated embryos showed expansion of the Tbx3expressing region in the AV canal over the boundary with ventricular myocardium.

Tbx 2 represses growth and maturation of the atrial and ventricular chambers $(10$, 11), and Tbx 2 deficiency results in ectopic expression of chamber-specific myocardial genes in the AV canal and malformation of the AV junction and base of the left ventricle $(11,19)$. In this issue of the JCI, detailed analysis by Aanhaanen and colleagues revealed that $\mathrm{Tbx} 2$-deficient mice on the mixed C57BL/6/129/ICR background manifested ventricular preexcitation and formation of left-sided accessory pathways equipped with fast conduction property (Figure 1D and ref. 2). Ectopic expression of high conductance gap junction and $\mathrm{Na}^{+}$ channel proteins in the left-sided AV canal contributed to fast conduction of the aberrant AV connections. In addition, myocardium at the epicardial side of the left AV canal was more proliferative, which may impede the inward migration of sulcus tissue and interrupt the formation of the annulus fibrosus. Since myocardium-specific inactivation of Tbx 2 was sufficient for the formation of accessory pathways, aberrant patterning of the AV canal as a consequence of Tbx 2 inactivation leads to the formation of the accessory pathways and malformation of the annulus fibrosus.

\section{Ventricular preexcitation in mice and humans}

As discussed above, accessory pathways must be the remnant products of diverse genetic and environmental etiologies, causing malformation of the AV canal and interruption of the annulus fibrosus. An important observation in the study by Aanhaanen et al. is that Tbx2 function in the AV canal is coupled to annulus fibrosus formation (2). In addition to restricting growth of the AV canal and thus spatially allowing inward migration of the epicardial derived sulcus tissue, Tbx2 was found to regulate normal patterning and maturation of the sulcus tissue, because the level of expression of several EPDC markers, including collagen 3a1, Wilms' tumor 1 homolog, and Tbx18, was markedly decreased in myocardium- specific Tbx2 mutant mice (2). It remains unknown how Tbx2 in the AV canal controls gene expression and cellular function in sulcus tissue, and this will be an important issue for future investigation. Given that remnant strands of AV myocardium can still be observed in otherwise normal hearts of quails and humans $(14,15)$, the presence of accessory pathways is not sufficient to explain the pathogenesis of ventricular preexcitation. Although the level of expression of several genes related to fast conduction was increased in the accessory pathways of Tbx2-deficient mice (2), a more comprehensive analysis of gene expression in the accessory pathways in these mice will provide clues as to how accessory pathways acquire their fast conduction property.

While Notch1-activated mice and Tbx2deficient mice share similar developmental defects, there are important differences in spatial and temporal patterns of accessory pathway formation. The accessory pathways in the Notch1-activated mice were most commonly epicardial and right-sided, but left-sided connections and myocardial cuffing of the coronary veins were also observed (1). Ventricular preexcitation was not recorded at birth but became apparent as the mice grew (1). In contrast, the accessory pathways in the Tbx2-deficient mice were predominantly left-sided, possibly because of compensation for Tbx 2 deficiency by $\mathrm{Tbx} 3$ in the right-sided region of the AV canal (2). Ventricular preexcitation was observed during late embryogenesis in myocardium-specific Tbx2-deficient mice (2). In humans, accessory pathways producing ventricular preexcitation are relatively smaller than those of Notch1activated mice and Tbx2-deficient mice and can be observed anywhere at the AV boundaries, but left-sided, right-sided, and septal connections are usually responsible for WPW syndrome (20). Ventricular contraction is stimulated by electrical impulses both through the AV node and the accessory pathway in human WPW syndrome, but the ventricle is completely preexcited without the involvement of the AV nodal conduction in Notch1-activated mice and Tbx2-deficient mice. Although Notch1-activated mice and Tbx2-deficient mice do not fully recapitulate the morphological and electrophysiological features of human WPW syndrome, they will be very helpful for enhancing understanding of the developmental mechanisms of accessory pathway formation that underlie ventricular preexcitation. 


\section{Conclusions and perspectives}

The current reports by Rentschler et al. and Aanhaanen et al. clearly demonstrate that accessory pathway formation underlying ventricular preexcitation arises as a consequence of erroneous development of the AV canal myocardium. A hierarchical network of transcription factors, including Tbx2/3/5, Nkx2.5, GATA4, and Msx2, governs normal development of the AV canal under the regulation of Notch and BMP signaling (10). Contrary to previous reports demonstrating that Notch signaling restricts BMP2 and $\mathrm{Tb} \times 2 / 3$ to the $\mathrm{AV}$ canal during early embryogenesis $(16,17)$, the expression level of BMP2 was unchanged in Notch1-activated mice (1). Our understanding of the pathophysiology of ventricular preexcitation will be broadened by further characterization of the genetic program for AV canal development.

\section{Acknowledgments}

This work was supported in part by grants from Japan Society for the Promotion of Science (KAKENHI 20390218, 21229010); Health and Labor Sciences Research grants (to I. Komuro and H. Akazawa); and grants from Astellas Foundation for Research on Metabolic Disorders, The Uehara Memorial Foundation, The Ichiro Kanehara Foundation, Mochida Memorial Foundation for Medical and Pharmaceutical Research, and Suzuken Memorial Foundation (to H. Akazawa).
Address correspondence to: Issei Komuro, Department of Cardiovascular Medicine, Osaka University Graduate School of Medicine, 2-2 Yamadaoka, Suita, Osaka 565-0871, Japan. Phone: 81.6.6879.3631; Fax: 81.6.6879.3639; E-mail: komuro-tky@ umin.ac.jp.

1. Rentschler $S$, et al. Notch signaling regulates murine atrioventricular conduction and the formation of accessory pathways. J Clin Invest. 2011;121(2):525-533.

2. Aanhaanen WTJ, et al. Defective Tbx2-dependent patterning of the atrioventricular canal myocardium causes accessory pathway formation in mice. J Clin Invest. 2011;121(2):534-544.

3. Blair E, et al. Mutations in the gamma(2) subunit of AMP-activated protein kinase cause familial hypertrophic cardiomyopathy: evidence for the central role of energy compromise in disease pathogenesis. Hum Mol Genet. 2001;10(11):1215-1220.

4. Gollob $\mathrm{MH}$, et al. Identification of a gene responsible for familial Wolff-Parkinson-White syndrome. N Engl J Med. 2001;344(24):1823-1831.

5. Arad M, Seidman CE, Seidman JG. AMP-activated protein kinase in the heart: role during health and disease. Circ Res. 2007;100(4):474-488.

6. Arad $M$, et al. Transgenic mice overexpressing mutant PRKAG2 define the cause of Wolff-Parkinson-White syndrome in glycogen storage cardiomyopathy. Circulation. 2003;107(22):2850-2856.

7. Sidhu JS, et al. Transgenic mouse model of ventricular preexcitation and atrioventricular reentrant tachycardia induced by an AMP-activated protein kinase loss-of-function mutation responsible for Wolff-Parkinson-White syndrome. Circulation. 2005;111(1):21-29.

8. Wessels A, Markman MW, Vermeulen JL, Anderson RH, Moorman AF, Lamers WH. The development of the atrioventricular junction in the human heart. Circ Res. 1996;78(1):110-117.

9. Kolditz DP, et al. Epicardium-derived cells in development of annulus fibrosis and persistence of acces- sory pathways. Circulation. 2008;117(12):1508-1517.

10. Hatcher CJ, Basson CT. Specification of the cardiac conduction system by transcription factors. Circ Res. 2009;105(7):620-630.

11. Aanhaanen WT, et al. The Tbx $2+$ primary myocardium of the atrioventricular canal forms the atrioventricular node and the base of the left ventricle. Circ Res. 2009;104(11):1267-1274.

12. Aanhaanen WT, et al. Developmental origin, growth, and three-dimensional architecture of the atrioventricular conduction axis of the mouse heart. Circ Res. 2010;107(6):728-736.

13. Tallini YN, et al. Imaging cellular signals in the heart in vivo: Cardiac expression of the high-signal Ca2+ indicator GCaMP2. Proc Natl Acad Sci U S A. 2006;103(12):4753-4758.

14. Kolditz DP, et al. Persistence of functional atrioventricular accessory pathways in postseptated embryonic avian hearts: implications for morphogenesis and functional maturation of the cardiac conduction system. Circulation. 2007;115(1):17-26.

15. Hahurij ND, et al. Accessory atrioventricular myocardial connections in the developing human heart: relevance for perinatal supraventricular tachycardias. Circulation. 2008;117(22):2850-2858.

16. Rutenberg JB, Fischer A, Jia H, Gessler M, Zhong TP, Mercola M. Developmental patterning of the cardiac atrioventricular canal by Notch and Hairy-related transcription factors. Development. 2006;133(21):4381-4390.

17. Kokubo H, Tomita-Miyagawa S, Hamada Y, Saga Y. Hesr1 and Hesr2 regulate atrioventricular boundary formation in the developing heart through the repression of Tbx2. Development. 2007;134(4):747-755.

18. Ma L, Lu MF, Schwartz RJ, Martin JF. Bmp2 is essential for cardiac cushion epithelial-mesenchymal transition and myocardial patterning. Development. 2005;132(24):5601-5611.

19. Harrelson $Z$, et al. Tbx 2 is essential for patterning the atrioventricular canal and for morphogenesis of the outflow tract during heart development. Development. 2004;131(20):5041-5052.

20. Anderson RH, Ho SY. Anatomy of the atrioventricular junctions with regard to ventricular preexcitation. Pacing Clin Electrophysiol. 1997;20(8 pt 2):2072-2076.

\section{Growing a tumor stroma: a role for granulin and the bone marrow}

Andrew Bateman

Endocrine Research Laboratory, Royal Victoria Hospital, Research Institute of the McGill University Health Centre, Montreal, Quebec, Canada.

\begin{abstract}
The tumor stroma is critical in cancer progression; understanding its formation is therefore important biologically and therapeutically. In this issue of the JCI, Elkabets et al. report on the generation of data in mice that lead them to propose that certain tumors can stimulate the growth of a second otherwise quiescent or indolent tumor in the same animal by stimulating stromal formation. Granulin-expressing $\mathrm{Sca}^{+} \mathrm{Kit}^{-}$hematopoietic progenitor cells in the bone marrow of the tumor host were required to mediate this effect. These data shed new light on the importance of the bone marrow in tumor growth and the role of granulin in carcinogenesis.
\end{abstract}

Conflict of interest: The author has declared equity in Neurodyn Inc.

Citation for this article: J Clin Invest. doi:10.1172/ JCI46088.

\section{Background: systemic tumor instigation}

The reactive (or desmoplastic) stroma is an aberrant fibrous tissue that surrounds can- cer cells (1). It is formed from fibroblasts, adipocytes, inflammatory cells, and vascular cells and is further characterized by the presence of myofibroblasts (1). Myofibroblasts display properties not usually associated with fibroblasts in healthy tissue, such as the expression of $\alpha \mathrm{SMA}$, and secrete high levels of matrix proteins such as collagen I (1). The molecular properties of tumor stroma are predictive of disease outcome (2), with the stromal cells, in particular the myofibroblasts, stimulating tumor growth, invasion, and metastasis (1). For example, molecular crosstalk between cancer cells 